\title{
Interactive comment on "Origin of the power-law exponent in the landslide frequency-size distribution” by Ahoura Jafarimanesh et al.
}

\section{Anonymous Referee \#2}

Received and published: 18 October 2018

This paper aims to provide a physical understanding regarding the factors controlling power-law exponent, which is widely observed in frequency-size distributions of landslides. Although the power-law relation is observed in a number of landslide inventories, the reasoning behind this relation and the factors controlling power-law exponent are still under discussion. Therefore, this paper addresses an interesting scientific question, and the paper has a fit to NHESS. I think this study could possibly be publishable with major revisions.

General comments:

Printer-friendly version

1. The paper is missing some key literature that aims to provide a physical explanation for the power-law distribution of landslides. For example, Liucci et al. (2017) (http://dx.doi.org/10.1016/j.geomorph.2017.04.017) introduced a model: "the model is
Discussion paper

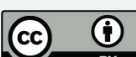


capable of reproducing the scaling behavior of real landslide areas and suggest that topography is a good candidate to explain their scale-invariance." On the other hand, Stark and Guzzetti (2009) (doi:10.1029/2008JF001008) and Frattini and Crosta (2013) (http://dx.doi.org/10.1016/j.epsl.2012.10.029 ) use geotechnical parameters to explain the power-law exponent of the landslide size distribution. For example, Frattini and Crosta (2013) argue that "the exponent of the power-law tail is controlled by both the topography and the depth profile of material strength." I think the authors should elaborate on these papers and indicate the points that they agree with these papers. The authors then need to indicate what is missing in the literature; what they argue differently than the previous studies.

2. The authors also need to indicate why exploring the factors controlling power-law exponent is important. There is one line in the abstract saying that "So far, the origin of the $\alpha$-value, critical to probabilistic hazard assessment, remains hypothetical." This may be enough for the abstract. However, in the introduction, they should explain it further because there is only one line at the end of the introduction saying that "This is of importance in the probabilistic hazard assessment of landslides to be able to extrapolate the size of larger potentially damaging events."

3. I am aware of the large literature examining the power-law relation that assumed to be valid for landslide frequency-size distribution. However, I think the authors should note that this is still an assumption. For example, in page 2, line 2, the authors indicated that "the frequency-size distribution (FSD), which, despite the variety of triggers, appears to systematically follow a power-law probability density function." I believe it is still up for debate whether that is, in fact, the case, especially if there is a physical mechanism responsible for deviation from power-law scaling. Therefore, it might be better to emphasize that this is what we observe most of the time. However, for example, Tanyas et al. (2018) (DOI: 10.1002/esp.4359) examine the validity of power-law relation using KS-test and show that "...six out of the 45 inventories have P-values lower than 0.1 ", so the power-law fit may not be a plausible hypothesis (?). Thus, the

Printer-friendly version Discussion paper

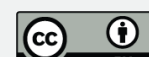


authors need to mention that it is not a fact, but a general observation.

4. The authors have a nice literature summary showing power-law exponents reported in the literature. However, there are still some missing papers. For example, ten Brink et al. (2006) (doi:10.1029/2006GL026125) analyzed sub-marine landslides. Tanyaş et al. (2018) (DOI: 10.1002/esp.4359) examined 45 landslide inventories and reported that power-law exponents of earthquake-triggered landslide inventories range from 1.8 to 3.7 .

5. I would like to be informed by the limitations of the model. This is a simplified model to understand a natural process. So, there must be some limitations, and I think the authors should list them in the manuscript. For example, in Table 2, some geotechnical parameters are presented and used to calculate FS in the modeling stage. This is quite a simplification, and I do not mean that the author can not use them but if you use them, then its limitations should be addressed as well. In this regard, somewhere before the Conclusion section, the authors should consider adding a Discussion section to discuss these issues. The things I mentioned in the previous item (4) can also be discussed in a Discussion section.

6. There are some basic concepts mentioned in the manuscript without giving enough background information. For example, what is Df? What is the Hurst exponent? Beyond its role in equation (1) what power-law exponent stands for? What is the physical meaning of having larger or smaller beta or Df, etc.? Similarly, what do you mean by landslide propagation phase? I think the authors should give some background about such terms in the introduction section, so the reader can understand why you are telling about, for example, propagation phase, and what it refers to?

7. In terms of the structure, I think it would be better the paper has a clear methodology, results and discussion sections. In the current version the discussion section is missing, and I think it is a must and you should add a discussion section (please see my comments below). Also, in the result section, there are some parts that need

Printer-friendly version

Discussion paper 
to be moved to the method section. For example, in page 5, line 25, authors say that "To validate our model based on FSD statistics (section 3.2), we first update the review made by Van Den Eeckhaut et al. (2007)." Similarly, in page 6, line 7, the authors say that "We first evaluated $\alpha$ at different steps of the landslide process: (i)....(ii)." These are just examples for the parts that need to be moved to the methodology section.

8. The authors used the mean values to validate their approach: (In page 7, line 19) "After revisiting the literature, we updated the observed power-law exponent distribution to $\alpha=2.21 \pm 0.53$ and validated our $20 \mathrm{LSgCA}$ by obtaining a similar distribution in simulations with $\alpha=2.17 \pm 0.49$." Also, for example, in figure 5 authors show that the range of estimated and real exponents match. The question in my mind then why do not the authors select one or two landslides affected area that authors have landslide inventories to estimate those particular cases. For example, the authors can examine the sites of which landslide frequency-size distributions are presented in Figure 1. Alternatively, some other sites that the authors have access to their environmental conditions (e.g., lithology, hydrology, etc.) to estimate the parameters need to run the FS equation. Then the authors would make an event-based validation. This is just an idea, and I am wondering it is possible or not.

Line by line comments:

Page 2, Line 9. "However all those models provide similar estimates of $\alpha$, as illustrated in Fig. 1". I do not agree with this statement; please take a look at my general comment (4). There are cases that we can observe different power-law exponents.

Page 2, Line 23. "but only considered the area of the landslide initiation phase, not of the landslide itself." Please elaborate further, not so clear what you mean by this sentence. What do you mean by "area of the landslide initiation phase" and "landslide itself."

Page 2, Line 25. "Piegari et al., (2006; 2009) only obtained reasonable $\alpha$ estimates by allowing arbitrary parameter variations to match the data (see discussion in Hergarten,

Printer-friendly version

Discussion paper 
2013)" What for you mean by "reasonable"? The typical range of previously observed values for landslide inventories (1.4-3.4), which have a central tendency of around 2.32.5 (Van Den Eeckhaut et al., 2007; Stark and Guzzetti, 2009). So, we have power-law exponents range from 1.4-3.4, then what is reasonable. It might be better if you give a further explanation instead of directing us to the discussion section of Hergarten, 2013.

Page 3, Line 29. "An FS map can then be produced that depends mainly on the spatial distribution of $\theta$, i.e., on the topography." This is not clear to me; please explain a bit. You are presenting us FS equations where FS depends on many variables, and then you are saying that FS mainly depends on $\theta$. Why?

Page 3, Line 4. "As investigated in section 3, we do not expect the use of different FS models, nor different triggers, to have an impact on the landslide FSD $\alpha$-value since they only impact the spatial extent of the landslide initiation zones defined by the thresholds FS $\leq 1$ or $\mathrm{DN} \geq 15 \mathrm{~cm}$." This is not clear to me; please elaborate further.

Page 5, Line 23. " $\alpha=1.5 \alpha \mathrm{V}$ when the landslide size is volume $\mathrm{V}$ instead of area $\mathrm{A}$ (with $\mathrm{V} \sim \mathrm{A} 3 / 2$ )." You need to give a reference here. You can use various scaling exponent in the equation between landslide volume and area $\left(\mathrm{V}=\alpha \mathrm{A}^{\wedge} E^{\wedge} \mathrm{c}\right)$. Please give a look at the literature (e.g., Guzzetti et al., 2009; Klar et al., 2011; Larsen et al., 2010) and let us know the reference you used.

Page 6, Line 1. "We finally obtain the distribution $\alpha=2.21 \pm 0.53$ " Please rephrase your sentence and indicate that this is the average value you calculated from the literature survey and please also refer to Figure 3.

Page 6, Line 2. "We ran simulations in which the ground parameters were drawn randomly from the range of values presented in Table 2." I think Table 2 deserves more explanation. You listed the geotechnical material properties of four different rock types. However, in a slope stability problem, the shear strength parameters are generally controlled by rock mass parameters, not the rock material parameters (e.g., Hoek and Brown, 1980). If the slope failure occurs along a discontinuity surface then the shear

Printer-friendly version

Discussion paper 
strength parameters of the discontinuity surface are used (e.g., Barton and Choubey, 1977). Please elaborate further why did you use these material properties and considered only these four rock types.

Minor comments:

Please go through your references and drop the "," coming after et al. (like the example I gave below) Page2, line 26. Guthrie et al., (2008)

Figure 1. The x-axis does not refer landslide count; it should be landslide frequency that is count per interval. Moreover, please indicate its unit $\left(\mathrm{m}^{\wedge}-2\right)$

Figure 2. You do not say any word about liquefaction that you indicated in this figure. I think you may better describe this figure and the supplementary material you provided.

Figure 3. Please make your labels a bit bigger and readable. Moreover, please use the correct symbol for the power-law exponent in the figure.

Figure 5. Please put a label for the y-axis.

Figure 6. Please put a label for the y-axis.

References Barton N, Choubey V. 1977. The shear strength of rock joints in theory and practice. Rock mechanics 10: 1-54. DOI: 10.1007/bf01261801 Guzzetti F, Ardizzone F, Cardinali M, Rossi M, Valigi D. 2009. Landslide volumes and landslide mobilization rates in Umbria, central Italy. Earth and Planetary Science Letters 279: 222-229. DOI: https://doi.org/10.1016/j.epsl.2009.01.005 Hoek E, Brown ET. 1980. Empirical strength criterion for rock masses. Journal of Geotechnical and Geoenvironmental Engineering 106: 1013-1035 Klar A, Aharonov E, KalderonâĂŘAsael B, Katz O. 2011. Analytical and observational relations between landslide volume and surface area. Journal of Geophysical Research: Earth Surface (2003-2012) 116. DOI: 10.1029/2009JF001604 Larsen IJ, Montgomery DR, Korup O. 2010. Landslide erosion controlled by hillslope material. Nature Geoscience 3: 247-251. DOI: 10.1038/ngeo776

Printer-friendly version

Discussion paper 
Interactive comment on Nat. Hazards Earth Syst. Sci. Discuss., https://doi.org/10.5194/nhess2018-167, 2018.

\section{NHESSD}

Interactive

comment 